\title{
Oncogenic role of abnormal spindle-like microcephaly-associated protein in lung adenocarcinoma
}

\author{
JIANG WANG $^{1,2}$, JINGHUI LIANG $^{1 *}$, HAIXIA LI $^{3 *}$, JINGYI HAN $^{1 *}$, JIN JIANG $^{1 *}$, \\ YONGMENG LI ${ }^{1}$, ZITONG FENG ${ }^{1}$, RENCHANG ZHAO ${ }^{1}$ and HUI TIAN ${ }^{1}$ \\ ${ }^{1}$ Department of Thoracic Surgery, Qilu Hospital, Cheeloo College of Medicine, Shandong University, Jinan, Shandong 250012; \\ ${ }^{2}$ Department of Thoracic Surgery, Weifang People's Hospital, Weifang, Shandong 261000; ${ }^{3}$ Department of Physiology and \\ Pathophysiology, School of Basic Medical Sciences of Shandong University, Jinan, Shandong 250012, P.R. China
}

Received December 16, 2020; Accepted March 10, 2021

DOI: $10.3892 /$ ijo.2021.5203

\begin{abstract}
Lung adenocarcinoma (LUAD) is a common malignant cancer worldwide. It is urgent to explore its underlying molecular mechanism and identify novel diagnostic biomarkers. Abnormal spindle-like microcephaly (ASPM) has recently received considerable attention due to its function in tumor progression. However, its role in LUAD is unclear. The present study aimed to explore the clinical role of ASPM in LUAD. Seven pairs of LUAD and adjacent normal tissues were collected to identify potential LUAD biomarkers using transcriptome sequencing. The association between ASPM expression and LUAD progression was evaluated using bioinformatics analysis and data obtained from clinical specimens. Using small interfering RNA technology, the function of ASPM was analyzed in the LUAD H1299 and A549 cell lines. Transcriptional profiling of ASPM-deficient H1299 cells was then performed to determine the downstream targets of ASPM Using databases and clinical specimens, it was revealed that ASPM expression was frequently elevated in LUAD tissues, and this upregulation was highly associated with LUAD progression. ASPM served as an oncogenic regulator of LUAD cell proliferation and metastasis. Mechanistically, ASPM facilitated epithelial-mesenchymal transition (EMT) via the
\end{abstract}

Correspondence to: Dr Hui Tian, Department of Thoracic Surgery, Qilu Hospital, Cheeloo College of Medicine, Shandong University, Wenhuaxi Street 44, Jinan, Shandong 250012, P.R. China

E-mail: tianhuiql@126.com

${ }^{*}$ Contributed equally

Abbreviations: LUAD, lung adenocarcinoma; ASPM, abnormal spindle-like microcephaly; EMT, epithelial-mesenchymal transition; CCK-8, Cell Counting Kit-8; EDU, 5-Ethynyl-2'-deoxyuridine; TCGA, The Cancer Genome Atlas; IHC, immunohistochemistry; RT-qPCR, reverse transcription-quantitative PCR; OS, overall survival; MAPK, mitogen-activated protein kinase; RAP1, Ras-proximate-1; CAM, cell adhesion molecule; GO, Gene Ontology

Key words: abnormal spindle-like microcephaly, lung adenocarcinoma, EMT, PI3K/AKT
PI3K/AKT signaling pathway and $740 \mathrm{Y}-\mathrm{P}$, an activator of this pathway, restored the migratory ability of ASPM-knockdown LUAD cells. The current study identified ASPM as an independent prognostic biomarker of LUAD that served an important oncogenic role in regulating LUAD cell metastasis by promoting EMT via the PI3K/AKT signaling pathway. Targeting ASPM may therefore be a therapeutic strategy for treating LUAD.

\section{Introduction}

In 2020, the morbidity of lung cancer ranked second and its mortality ranked first in both females and males worldwide $(1,2)$. Lung adenocarcinoma (LUAD) is the major type of lung cancer. Therapeutic options include surgery and treatment using radiotherapy, adjuvant chemotherapy, targeted drugs and immunotherapy (3). However, the 5-year survival rate in patients with LUAD remains low, and the risk of recurrence is high due to the metastatic nature of the tumor (4). Therefore, further progress is required to identify effective prognostic biomarkers in high-risk patients with LUAD and to elucidate the molecular mechanisms underlying LUAD development.

Abnormal spindle-like microcephaly (ASPM) protein was first identified as a microcephaly-associated protein that controls spindle architecture (5), which is essential for neurogenesis and brain size determination $(6,7)$. Additionally, ASPM participates in the process of symmetric stem cell division by promoting cyclin E ubiquitination (8). Previous studies have suggested that ASPM regulates microtubule degradation at the mitotic spindle pole by promoting katanin-mediated cleavage of dynamic microtubules $(9,10)$. In particular, ASPM exhibits both cytoplasmic and nuclear localization during interphase, and its cytoplasmic expression levels vary significantly among different types of tumor (11-13). This suggests that ASPM may have diverse biological functions in malignant tissues. Furthermore, ASPM is an oncofetal protein that is overexpressed in numerous malignant neoplasms, including pancreatic ductal adenocarcinoma (14), prostate cancer (13), ovarian cancer (15), hepatocellular carcinoma (16) and bladder cancer (17). The oncogenic function of ASPM has been demonstrated to be involved in regulation of cell cycle completeness and stem cell properties (8). However, the role of ASPM in 
LUAD remains unclear. Therefore, the present study aimed to explore the clinical role of ASPM in LUAD.

\section{Materials and methods}

Human tissue samples and cell lines. A total of 109 fresh paired normal and pathologically confirmed LUAD tumor tissues (7 pairs used for RNA-sequencing, 94 pairs for tissue microarray and 8 pairs for western blotting) were obtained from Qilu Hospital (Shandong University, Jinan, China) between September 2014 and June 2020. The normal tissues ( $\geq 5 \mathrm{~cm}$ from tumour tissues) were obtained from dissected lung tissues. The median age of patients was 62 years and the age range was 30-84 years among all 109 patients, with 59 males and 50 females in total. Both overall survival (OS) and progression-free survival (PFS) were analyzed. TNM stages were established according to the American Joint Committee on Cancer 8th edition (18). Patients did not receive any chemotherapy or radiotherapy before surgery. Patients provided written informed consent for the use of their samples in the study. All pathological results were confirmed by pathology. Complete clinicopathological and follow-up data for the 94 paired LUAD samples were available. All experiments were approved and supervised by the Medical Ethics Committee of Qilu Hospital of Shandong University (approval no. KYLL-2016-097).

Normal lung epithelial cells (HBE) and human lung cancer cell lines (HCC827, A549, H157, H1299 and H1975) were obtained from The Cell Bank of Type Culture Collection of The Chinese Academy of Sciences. The H157 cell line was authenticated by STR profiling. Cells were cultured in RPMI 1640 medium (cat. no. CF0001; Shandong Sparkjade Biotechnology Co., Ltd.) supplemented with 10\% FBS (cat. no. 04-001-1A; Biological Industries) and $1 \%$ antibiotic-antimycotic agents at $37^{\circ} \mathrm{C}$ in a $5 \% \mathrm{CO}_{2}$ cell culture incubator.

Cell transfection using small interfering (si)RNA. siRNA silencing was performed by transient transfection using Lipofectamine ${ }^{\circledR} 2000$ (Thermo Fisher Scientific, Inc.). Cells were seeded in a 6-well plate at a density of $2 \times 10^{5}$ cells/well. Once the cells had adhered, they were transfected with $5 \mu \mathrm{l}$ siRNA $(20 \mu \mathrm{M})$ or negative control (NC) for $8 \mathrm{~h}$ at $37^{\circ} \mathrm{C}$. Cells were incubated with the transfection mixture for 6-8 h, after which the medium was replaced with fresh RPMI 1640 medium. siRNA molecules against ASPM were purchased from Shanghai GenePharma Co., Ltd., with a scrambled siRNA used as the NC. The siRNA sequences were as follows: NC sense, 5'-UUCUCCGAACGUGUCACGUTT-3' and antisense, 5'-ACGUGACACGUUCGGAGAATT-3'; siRNA1 sense, 5'-CCCACCACCACUGUAUGAUTT-3' and antisense, 5'-AUCAUACAGUGGUGGUGGGTT-3'; and siRNA2 sense, 5'-GCUUGCAAUACAGCAAUA ATT-3' and antisense, 5'-UUAUUGCUGUAUUGCAAGCTT-3'.

Recombinant vector construction and cell transfection. The interference vector, pGMLV-SC5 (iGene Biotechnology Co., Ltd) was linearized using double restriction digestion and BamHI and EcoRI (Fermentas; Thermo Fisher Scientific, Inc.), purified using a Gel-Spin DNA Extraction kit (Tiangen Biotech Co., Ltd.) and then ligated with a short hairpin (sh)
RNA against ASPM (shASPM) or a negative control shRNA (shNC) (both iGene Biotechnology Co., Ltd) using T4 ligase (Fermentas; Thermo Fisher Scientific, Inc.). DH5 $\alpha$ competent cells (Tiangen Biotech Co., Ltd.) were mixed with the constructed vectors, which were then harvested using DNA Gel/PCR Purification Maxiprep kit (Generay Biotech Co., Ltd.). All the vectors were then transfected into 293T cells (The Cell Bank of Type Culture Collection of The Chinese Academy of Sciences) using the HG transgene reagent (iGene Biotechnology Co., Ltd) according to the manufacturer's instructions. High titers $\left(>10^{8}\right.$ transforming units $\left./ \mathrm{ml}\right)$ of the concentrated lentivirus solutions were harvested from the supernatant. The shRNAs were diluted to $0.1 \mathrm{nmol}$ for the downstream reaction, and the transfected cells were passaged to at least 5 generations (transfected after $24 \mathrm{~h}$ of culturing). Stable ASPM-knockdown H1299 and A549 cell lines were infected with lentivirus (MOI, 10 and 20, respectively) for $72 \mathrm{~h}$ and then selected with $1 \mathrm{mg} / \mathrm{ml}$ puromycin for $\sim 1$ week. After selection, the cells were used for further experiments.

Cell Counting Kit-8 (CCK-8) and 5-Ethynyl-2'-deoxyuridine (EDU) assay. Cell proliferation was measured using the CCK-8 (Beyotime Institute of Biotechnology) and EDU (Guangzhou RiboBio Co., Ltd.; cat. no. C10310) assays, according to the manufacturer's instructions. The cells were seeded in 96-well plates. Cell numbers were counted every $24 \mathrm{~h}$ after cell adhesion lasting for 4 days. The cells were incubated in CCK- 8 reagent for $2 \mathrm{~h}$. Subsequently, the absorbance was measured at a wavelength of $450 \mathrm{~nm}$ using a microplate reader (Bio-Rad Laboratories, Inc.). The experiment was repeated three times independently.

For EDU analysis, an EDU assay kit (cat. no. C10310-1; Guangzhou RiboBio Co., Ltd.) was used following the manufacturer's protocol. Cells were cultured in RPMI 1640 medium containing EDU for $4 \mathrm{~h}$ at $37^{\circ} \mathrm{C}$. Subsequently, cells were fixed with $4 \%$ paraformaldehyde at room temperature for $60 \mathrm{~min}$, after removing EDU-containing medium. After washing by penetrant (0.5\% TritonX-100 in PBS) and incubating on a decolorizing shaker for $10 \mathrm{~min}$, add Apollo staining reaction solution to each well lasting for $30 \mathrm{~min}$ on a decolorizing shaker in the dark at room temperature. Then Hoechst reaction solution was added to each well. After incubating for $30 \mathrm{~min}$ on a decolorizing shaker in the dark at room temperature, discard the staining reaction solution. After PBS washing, images were acquired using a fluorescence microscope (magnification, $\mathrm{x} 400$ ).

Wound healing, migration and invasion assay. For the wound healing assay, transfected and control cells were separately plated in 6-well plates. After the cells were completely attached, a sterile tip was used to draw straight lines on the bottom of the plate. After the scratch was made, the original medium was replaced by medium without FBS. The cells were washed with PBS, and the initial wound size was recorded with a light microscope (Olympus Corporation; magnification, $\mathrm{x} 50$ ). After incubation for $36 \mathrm{~h}$ at $37^{\circ} \mathrm{C}$, the current wound size was recorded. The wound closure percentage was calculated using the following formula: [1-(current wound size/initial wound size)] x100.

Cell migration or invasion were performed using Transwell chambers coated without or with Matrigel, respectively (both 
Corning, Inc.). Matrigel was added to Transwell chambers at $4^{\circ} \mathrm{C}$ and then incubated at $37^{\circ} \mathrm{C}$ for $30 \mathrm{~min}$. In brief, cells $\left(1 \times 10^{5}\right)$ were seeded into the upper chamber for adhesion with or without a monolayer of 5\% Matrigel and then serum-starved for $6 \mathrm{~h}$. The chambers were then placed in 24-well plates containing medium with $10 \%$ FBS in the bottom chambers. After $24 \mathrm{~h}$ at $37^{\circ} \mathrm{C}$, the cells adhering to the lower surface were fixed with methanol at $4^{\circ} \mathrm{C}$ for $1 \mathrm{~h}$ and stained with a $0.1 \%$ crystal violet solution at room temperature for $1 \mathrm{~h}$ (Sigma-Aldrich; Merck KGaA). The number of cells on the membrane was counted from five randomly selected visual fields with a fluorescence microscope (Olympus Corporation; magnification, $\mathrm{x} 200)$.

Transcriptome sequencing and bioinformatics analysis. Transcriptional sequencing of cells and tissues was performed by Hangzhou Lianchuan Biotechnology Co., Ltd. (https://www. omicstudio.cn/index). Total RNA was isolated from cell lines and tissues using the RNeasy Mini kit (Qiagen $\mathrm{GmbH}$ ). Related data were uploaded in the Gene Expression Omnibus (GEO) (http://www.ncbi.nlm.nih.gov/geo) dataset GSE140797 (19). The gene expression levels and clinical data of patients with LUAD from The Cancer Genome Atlas (TCGA) were downloaded from UCSC Xena Browser (https://xenabrowser. net/). The gene expression levels and clinical data of patients with LUAD from the GEO database were obtained using the GSE19804 (20), GSE116959 (21), GSE31210 (22) and GSE68465 (23) datasets (https://www.ncbi.nlm.nih.gov/gds). The volcano plot representing differentially expressed genes in LUAD and normal tissues was performed using R software (v3.6.1; www.r-project.org) according to $\mathrm{P}<0.05$ and fold-change $>2$. Kyoto Encyclopedia of Genes and Genomes (KEGG) and Gene Ontology (GO) enrichment analysis were performed using $\mathrm{R}$ software. $\mathrm{P}<0.05$ was defined as a significant enrichment analysis result $(24,25)$.

Immunohistochemistry $(I H C)$. Tissue sections fixed with $4 \%$ paraformaldehyde at $4^{\circ} \mathrm{C}$ for $24 \mathrm{~h}$ and embedded into paraffin were cut into $4-\mu \mathrm{m}$-thick sections and dried at room temperature for $24 \mathrm{~h}$. Paraffin-embedded LUAD and normal tissues sections were dewaxed with xylene and ethanol. Antigen retrieval was performed by incubation with sodium citrate buffer $\left(\mathrm{pH} 6.0\right.$ ) at $95^{\circ} \mathrm{C}$ for $15 \mathrm{~min}$. Sections were then blocked for endogenous peroxidase using $3 \% \mathrm{H}_{2} \mathrm{O}_{2}$ for $15 \mathrm{~min}$ at room temperature, washed by PBS for $5 \mathrm{~min}$ at room temperature and blocked in $10 \%$ goat serum (Beijing Solarbio Science \& Technology Co., Ltd.) for $1 \mathrm{~h}$ at room temperature. After washing for three times, the tissue sections were incubated with primary antibodies against ASPM (1:200; Abcam; cat. no. 238106), IgG isotype control (1:1,000; Cell Signaling Technology, Inc.; cat. no. 3900), E-cadherin (1:400; Cell Signaling Technology, Inc.; cat. no. 14472), N-cadherin (1:400; Cell Signaling Technology, Inc.; cat. no. 13116) and Ki67 (1:400; Cell Signaling Technology, Inc.; cat. no. 9027) in a wet box at $4^{\circ} \mathrm{C}$ overnight. Subsequently, tissue sections were incubated with a secondary antibody (1:200; Abcam; cat. no. 6721) for $2 \mathrm{~h}$ at room temperature. After staining with $\mathrm{DAB}$ and $\mathrm{H} \& \mathrm{E}$ for $10 \mathrm{~min}$ at room temperature, tissue sections were observed and photographed under a light microscope (magnifications, $x 100$ ). The tissue sections were scanned using a scanner to be scored using the nuclei quant software (v3.1; 3DHISTECH Ltd.) according to previous studies $(26,27)$.

Western blotting. Protein was extracted from tissues and cells using lysis buffer (Thermo Fisher Scientific, Inc.) with protease and phosphatase inhibitors (Roche Diagnostics). A BCA kit (Shanghai Yeasen Biotechnology Co., Ltd.; cat. no. B68010) was used to determine the protein concentration. Protein extracts (20 $\mu \mathrm{g} /$ lane) were separated by $10 \%$ SDS-PAGE and transferred to PVDF membranes (EMD Millipore). After blocking with 5\% milk in TBS-Tween (TBST; 0.1\% Tween 20) for $1 \mathrm{~h}$ at room temperature, membranes were incubated with primary antibodies against ASPM (1:500; cat. no. sc-488883; Santa Cruz Biotechnology, Inc.), GAPDH (used as a reference protein; 1:1,000; Cell Signaling Technology, Inc.; cat. no. 5174), E-cadherin (1:1,000; Cell Signaling Technology, Inc.; cat. no. 14472), N-cadherin (1:1,000; Cell Signaling Technology, Inc.; cat. no. 13116), Snail (1:1,000; Cell Signaling Technology, Inc.; cat. no. 3879), PI3K p110 $\alpha$ (1:1,000; Cell Signaling Technology, Inc.; cat. no. 4249), phosphorylated (p) AKT (1:1,000; Cell Signaling Technology, Inc.; cat. no. 4060) and AKT (1:1,000; Cell Signaling Technology, Inc.; cat. no. 4691) overnight at $4^{\circ} \mathrm{C}$. Subsequently, the membranes were incubated with an HRP-conjugated secondary antibody (1:1,000; Cell Signaling Technology, Inc.; cat. no. 7074) at room temperature for $1 \mathrm{~h}$. After washing with TBST 3 times, the proteins on the membranes were visualized by enhanced chemiluminescence (EMD Millipore; cat. no. WBKLS0010). The bands were visualized using the ChemiDoc ${ }^{\mathrm{TM}}$ MP Imaging System (Bio-Rad Laboratories, Inc.). The gray value was calculated using ImageJ v2.4.1 (National Institutes of Health). All of the protein expression levels, except pAKT expression, were calculated relative to GAPDH protein expression. pAKT expression was calculated relative to AKT protein expression.

Reverse transcription-quantitative PCR (RT-qPCR). Total RNA was isolated from cell lines using the RNeasy Mini kit (Qiagen $\mathrm{GmbH}$ ) and cDNA was synthesized using the PrimeScript ${ }^{\circledR}$ RT Reagent Perfect Real Time kit (Takara Biotechnology Co., Ltd.) according to the manufacturer's protocol. The cDNA was subjected to qPCR using the LightCycler $^{\circledR} 480$ SYBR Green I Master (Roche Diagnostics) following the manufacturer's instructions. GAPDH was used to normalize the amount of cDNA between different samples. The thermocycling conditions were as previously described $(28,29)$ : Initial denaturation at $95^{\circ} \mathrm{C}$ for $10 \mathrm{~min}$, followed by 40 cycles of denaturation at $95^{\circ} \mathrm{C}$ for $15 \mathrm{sec}$, annealing at $60^{\circ} \mathrm{C}$ for $15 \mathrm{sec}$ and extension at $70^{\circ} \mathrm{C}$ for $20 \mathrm{sec}$. Data analysis was performed using the $2^{-\Delta \Delta \mathrm{Cq}}$ method for relative quantification (30). All of the gene expression levels were calculated relative to the GAPDH gene. Sequences of primers were as follows: ASPM forward, 5'-GGCCCTAGACAACCC TAACGA-3' and reverse, 5'-AGCTTGGTGTTTCAGAAC ATCA-3'; and GAPDH forward, 5'-GGAGCGAGATCCCTC CAAAAT-3' and reverse, 5'-GGCTGTTGTCATACTTCT CATGG-3'.

PI3K/AKT pathway activator. 740 Y-P was obtained from MedChemExpress. The transfected LUAD cells were further treated with $740 \mathrm{Y}-\mathrm{P}(25 \mu \mathrm{g} / \mathrm{ml})$ for $24 \mathrm{~h}$ at $37^{\circ} \mathrm{C}$. 

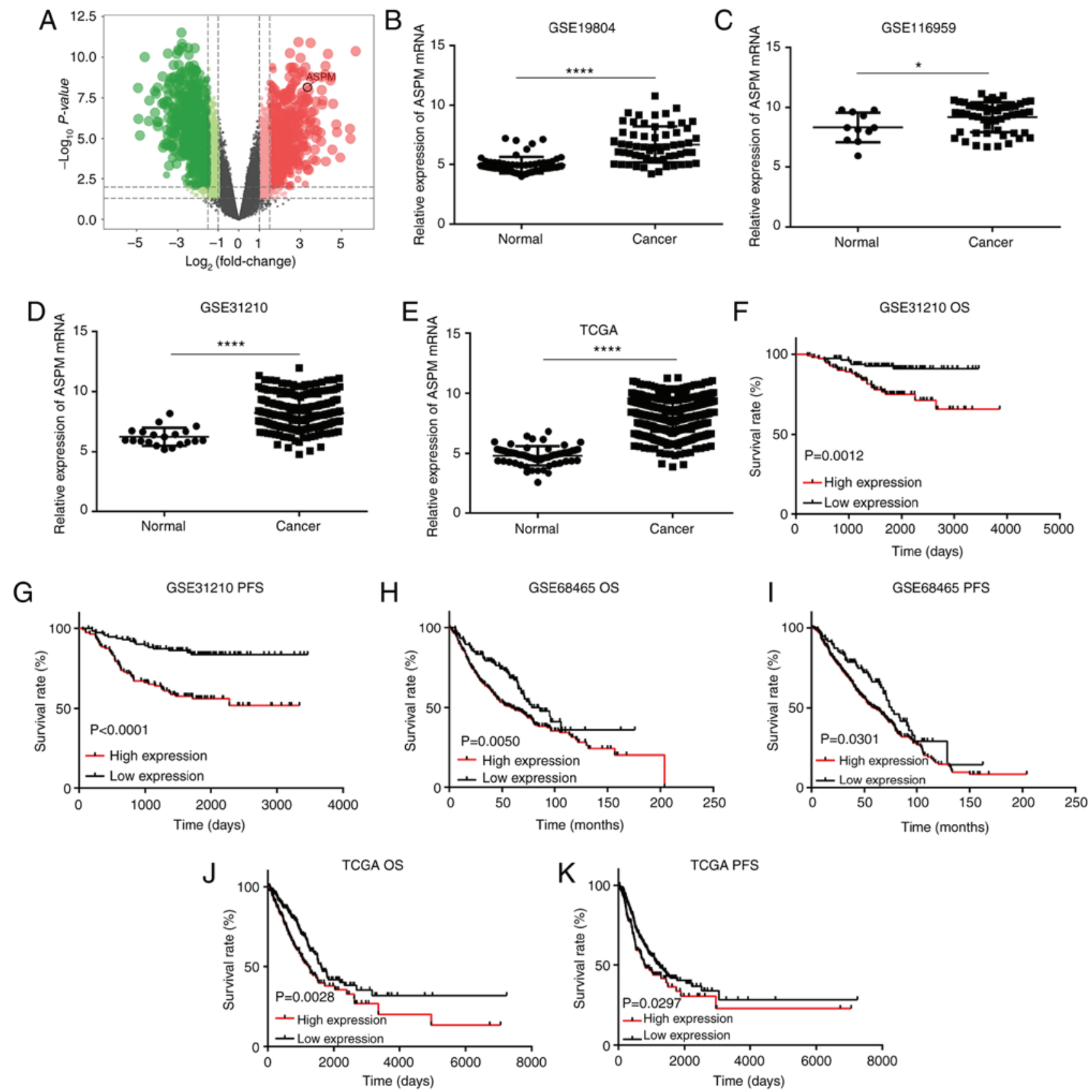

Figure 1. Bioinformatics analysis reveals that ASPM expression is upregulated in LUAD and predicts a poor prognosis in patients with LUAD. (A) Volcano plot of ASPM expression in 7 pairs of LUAD and tumor-adjacent tissues from transcriptome microarray sequencing. ASPM mRNA expression analysis in LUAD and non-tumor tissues in the Gene Expression Omnibus datasets (B) GSE19804, (C) GSE116959 and (D) GSE31210, and (E) TCGA database. (F) OS and (G) PFS of patients with LUAD were stratified by ASPM expression in GSE31210. (H) OS and (I) PFS of patients with LUAD were stratified by ASPM expression in GSE68465. (J) OS and (K) PFS of patients with LUAD were stratified by ASPM expression in TCGA database. " P $<0.05$; ${ }^{* * * * *} \mathrm{P}<0.0001$. LUAD, lung adenocarcinoma; ASPM, abnormal spindle-like microcephaly; TCGA, The Cancer Genome Atlas; OS, overall survival; PFS, progression-free survival.

Animal experiments. All animal experiments were approved by the Medical Ethics Committee of Shandong University (approval no. KYLL-2016-097). A total of 18 male nude BalB/c mice (age, 6-8 weeks old; weight, 20-22 g) were purchased from Beijing Vital River Laboratory Animal Technology Co., Ltd. Animals were housed in polycarbonate standard mouse cages. Housing conditions were kept constant at a temperature of $18-21^{\circ} \mathrm{C}$ and humidity of $40-70 \%$, with a 12 -h light/dark cycle. Pelleted mouse food and water was provided ad libitum. For the in vivo proliferation assay, $\sim 1 \times 10^{6} \mathrm{H} 1299$ cells (transfected with shASPM or shNC) in $100 \mu$ l PBS were subcutaneously injected into the left oxter of nude mice ( $\mathrm{n}=5 \mathrm{mice} / \mathrm{group}$ ). After 7 days, the first measurements of the length and width of tumors were made by caliper, and tumor growth was measured for 17 days after this point. After the tumor emerged, tumor volume was measured every three days, and tumor growth curves were calculated and compared. For the in vivo metastasis assay, $3 \times 10^{5} \mathrm{H} 1299$ cells (transfected with shASPM or control lentivirus) in $100 \mu \mathrm{l}$ PBS were injected into the caudal vein of each nude mice ( $n=4$ mice/group). After 7 weeks, the mice were sacrificed by decapitation under anesthesia with phenobarbital $(100 \mathrm{mg} / \mathrm{kg})$ intraperitoneal injection and the 
Table I. Association between ASPM expression and clinicopathological features in patients with lung adenocarcinoma from the GSE68465 dataset $(n=436)$.

\begin{tabular}{|c|c|c|c|c|}
\hline \multirow[b]{2}{*}{ Characteristics } & \multirow[b]{2}{*}{ All cases, n (\%) } & \multicolumn{2}{|c|}{ ASPM expression, $\mathrm{n}$} & \multirow[b]{2}{*}{ P-value } \\
\hline & & Low expression $(n=219)$ & High expression $(n=217)$ & \\
\hline Sex & & & & 0.0699 \\
\hline Male & $220(50.5)$ & 101 & 119 & \\
\hline Female & $216(49.5)$ & 118 & 98 & \\
\hline $\operatorname{Age}^{\mathrm{b}}$ & & & & 0.7746 \\
\hline$<65$ & $208(47.7)$ & 106 & 102 & \\
\hline$\geq 65$ & $228(52.3)$ & 113 & 115 & \\
\hline pT status & & & & $<0.0001$ \\
\hline $\mathrm{T} 1$ & $148(33.9)$ & 94 & 54 & \\
\hline $\mathrm{T} 2$ & $248(56.9)$ & 116 & 132 & \\
\hline $\mathrm{T} 3$ & $28(6.4)$ & 7 & 21 & \\
\hline $\mathrm{T} 4$ & $12(2.8)$ & 2 & 10 & \\
\hline pN status & & & & 0.0129 \\
\hline NO & $296(67.9)$ & 156 & 140 & \\
\hline $\mathrm{N} 1$ & $79(18.1)$ & 43 & 36 & \\
\hline $\mathrm{N} 2+\mathrm{N} 3$ & $61(14.0)$ & 20 & 41 & \\
\hline TNM stage & & & & 0.0001 \\
\hline I & $112(25.7)$ & 73 & 39 & \\
\hline II & $255(58.5)$ & 123 & 132 & \\
\hline III+IV & $69(15.8)$ & 23 & 46 & \\
\hline Differentiation & & & & $<0.0001$ \\
\hline Well & $60(13.8)$ & 58 & 2 & \\
\hline Medium & $209(47.9)$ & 112 & 97 & \\
\hline Poor & $167(38.3)$ & 49 & 118 & \\
\hline Event & & & & 0.0004 \\
\hline Alive & $202(46.3)$ & 120 & 82 & \\
\hline Dead & $234(53.7)$ & 99 & 135 & \\
\hline
\end{tabular}

${ }^{\text {aPearson's }} \chi^{2}$ test or Fisher's exact test was used for statistical analysis. ${ }^{\mathrm{b}}$ Age was divided based on the median age at diagnosis of 65 years. ASPM, abnormal spindle-like microcephaly; pT, pathological tumor; pN, pathological lymph node.

number of nodules in the lungs was confirmed by hematoxylin and eosin (H\&E) staining and counted.

$H \& E$ staining. Tumor and lung tissues from mice were fixed in $4 \%$ paraformaldehyde at $4^{\circ} \mathrm{C}$ for $24 \mathrm{~h}$, embedded in paraffin and cut into $10-\mu \mathrm{m}$-thick sections. The sections were deparaffinized in xylene, rehydrated in serially diluted ethanol and stained with H\&E (Sigma-Aldrich; Merck KGaA) at $4^{\circ} \mathrm{C}$ for $10 \mathrm{~min}$. Representative photomicrographs were captured using a light microscope (Olympus Corporation; magnifications, $\mathrm{x} 100$ and $\mathrm{x} 400$ ).

Statistical analysis. Statistical analysis was performed using R software (R version 3.6.1) and GraphPad Prism 6.0 (GraphPad Software, Inc.). The median cut-off value was used to separate high- and low-expression groups for Kaplan-Meier analysis. X-title software (v1.0.4; http://kinzler.com/me/xtitle) was used to select a suitable cut-off value to avoid crossover issues. For comparisons, unpaired and paired Student's t-test (two-sided),
Pearson's $\chi^{2}$ test, Kaplan-Meier survival analysis with log-rank test, and Fisher's exact test were performed as indicated. One-way ANOVA was used to compare the differences among $>2$ groups followed by Tukey's multiple comparisons post-hoc test. Univariate and multivariate Cox proportional-hazards regression analyses were performed to evaluate factors affecting survival. Data are presented as the mean \pm SD. Each experiment was performed in triplicate. $\mathrm{P}<0.05$ was considered to indicate a statistically significant difference.

\section{Results}

Bioinformatics analysis reveals upregulated ASPM expression and predicts a poor prognosis in patients with LUAD. First, seven pairs of frozen LUAD and adjacent normal tissues were collected to perform transcriptome analysis to screen for potential biomarkers (GSE140797). According to this analysis, it was revealed that ASPM mRNA expression was significantly increased in LUAD tissues compared with that 
Table II. Association between ASPM expression and clinicopathological features in patients with lung adenocarcinoma in The Cancer Genome Atlas database $(\mathrm{n}=337)$.

ASPM expression, $\mathrm{n}$

\begin{tabular}{|c|c|c|c|c|}
\hline & & & & \\
\hline Characteristics & All cases, $\mathrm{n}(\%)$ & Low expression $(n=168)$ & High expression $(n=169)$ & P-value \\
\hline Sex & & & & 0.3841 \\
\hline Male & $166(49.3)$ & 87 & 79 & \\
\hline Female & $171(50.7)$ & 81 & 90 & \\
\hline $\mathrm{Age}^{\mathrm{b}}$ & & & & 0.5852 \\
\hline$<66$ & $162(48.1)$ & 78 & 84 & \\
\hline$\geq 66$ & 175 (51.9) & 90 & 85 & \\
\hline pT status & & & & 0.0135 \\
\hline $\mathrm{T} 1$ & $102(30.3)$ & 64 & 38 & \\
\hline $\mathrm{T} 2$ & $189(56.1)$ & 82 & 107 & \\
\hline $\mathrm{T} 3$ & $28(8.3)$ & 15 & 13 & \\
\hline $\mathrm{T} 4$ & $18(5.3)$ & 7 & 11 & \\
\hline pN status & & & & 0.0630 \\
\hline No & $212(62.9)$ & 116 & 96 & \\
\hline N1 & $72(21.4)$ & 29 & 43 & \\
\hline $\mathrm{N} 2+\mathrm{N} 3$ & $53(15.7)$ & 23 & 30 & \\
\hline pM status & & & & 0.0221 \\
\hline M0 & $316(93.8)$ & 163 & 153 & \\
\hline M1 & $21(6.2)$ & 5 & 16 & \\
\hline TNM stage & & & & 0.0056 \\
\hline I & $172(51.1)$ & 100 & 72 & \\
\hline II & $83(24.6)$ & 36 & 47 & \\
\hline III & $61(18.1)$ & 27 & 34 & \\
\hline IV & $21(6.2)$ & 5 & 16 & \\
\hline Event & & & & 0.0110 \\
\hline Alive & $202(59.9)$ & 113 & 89 & \\
\hline Dead & $137(40.1)$ & 57 & 80 & \\
\hline
\end{tabular}

${ }^{\text {aPearson's }} \chi^{2}$ test or Fisher's exact test was used for statistical analysis. ${ }^{\mathrm{b}}$ Age was divided based on the median age at diagnosis of 66 years. ASPM, abnormal spindle-like microcephaly; pT, pathological tumor; pN, pathological lymph node; pM, pathological metastasis.

in adjacent normal tissues (Fig. 1A). To confirm this result, the GEO LUAD datasets (GSE19804, GSE116959 and GSE31210) and TCGA LUAD dataset were analyzed via bioinformatics analysis. Similarly, ASPM mRNA expression was significantly upregulated in LUAD tissues compared with that in normal lung tissues (Fig. 1B-E).

Subsequently, the association between clinicopathological parameters and ASPM mRNA expression in patients with LUAD was analyzed. The GSE68465 dataset was used as the discovery cohort (Table I) and TCGA dataset as the validation cohort (Table II). ASPM expression was significantly associated with pathological tumor (pT) status (discovery cohort, $\mathrm{P}<0.0001$; validation cohort, $\mathrm{P}=0.0135$ ), TNM stage (discovery cohort, $\mathrm{P}=0.0001$; validation cohort, $\mathrm{P}=0.0056$ ) and survival state (discovery cohort, $\mathrm{P}=0.0004$; validation cohort, $\mathrm{P}=0.0110$ ) in both cohorts (Tables I and II). Additionally, ASPM expression was significantly associated with lymph node status $(\mathrm{P}=0.0129)$ and differentiation status $(\mathrm{P}<0.0001)$ in the discovery cohort, but not in the validation cohort, and with pM status in the validation cohort $(\mathrm{P}=0.0221)$ (Tables I and II).

Furthermore, Kaplan-Meier survival analysis indicated that patients with higher expression levels of ASPM exhibited a poorer survival rate both in OS and PFS (Fig. 1F-K; $\mathrm{P}<0.05)$ than those who had lower expression levels of ASPM both in the GEO and TCGA datasets. To further explore the prognostic significance of the mRNA expression levels of ASPM in LUAD, univariate and multivariate Cox proportional-hazards regression analyses were performed. Univariate Cox regression analysis revealed that ASPM expression [hazard ratio (HR), 1.49; 95\% CI, 1.147-1.936; $\mathrm{P}=0.003$ in discovery cohort; HR, 1.926; 95\% CI, 1.364-2.718; $\mathrm{P}<0.001$ in the validation cohort) was a significant negative prognostic factor for OS (Tables III and IV). Multivariate analysis revealed that ASPM expression was an independent prognostic factor of OS (HR, 1.323; 95\% CI, 1.007-1.739; $\mathrm{P}=0.045$ in the discovery cohort; HR, 1.725; $95 \% \mathrm{CI}, 1.219-2.441 ; \mathrm{P}=0.002$ in the validation cohort) 
Table III. Univariate and multivariate Cox regression analysis of different prognostic factors in patients with lung adenocarcinoma from the GSE68465 dataset.

\begin{tabular}{|c|c|c|c|c|c|c|c|}
\hline \multirow[b]{2}{*}{ Characteristics } & \multirow[b]{2}{*}{ Sample, n (\%) } & \multicolumn{3}{|c|}{ Univariate analysis $^{\mathrm{a}}$} & \multicolumn{3}{|c|}{ Multivariate analysis ${ }^{\mathrm{a}}$} \\
\hline & & HR & $95 \% \mathrm{CI}$ & P-value ${ }^{b}$ & HR & $95 \% \mathrm{CI}$ & P-value ${ }^{b}$ \\
\hline $\mathrm{Age}^{\mathrm{c}}$ & & 1.544 & $1.191-2.002$ & 0.001 & 1.571 & $1.210-2.040$ & $<0.001$ \\
\hline$\leq 65$ & $224(51.6)$ & & & & & & \\
\hline$>65$ & $210(48.4)$ & & & & & & \\
\hline Sex & & 1.436 & $1.107-1.863$ & 0.006 & 1.231 & $0.943-1.607$ & 0.127 \\
\hline Female & $215(49.5)$ & & & & & & \\
\hline Male & $219(50.5)$ & & & & & & \\
\hline ASPM expression & & 1.490 & $1.147-1.936$ & 0.003 & 1.323 & $1.007-1.739$ & 0.045 \\
\hline Low expression & $217(50.0)$ & & & & & & \\
\hline High expression & $217(50.0)$ & & & & & & \\
\hline T stage & & 1.652 & $1.376-1.983$ & $<0.001$ & 1.374 & $1.130-1.671$ & 0.001 \\
\hline $\mathrm{T} 1$ & $147(33.9)$ & & & & & & \\
\hline $\mathrm{T} 2$ & $248(57.1)$ & & & & & & \\
\hline $\mathrm{T} 3$ & $28(6.5)$ & & & & & & \\
\hline $\mathrm{T} 4$ & $11(2.5)$ & & & & & & \\
\hline $\mathrm{N}$ stage & & 2.012 & $1.710-2.368$ & $<0.001$ & 1.987 & $1.687-2.34$ & $<0.001$ \\
\hline N1 & $296(68.2)$ & & & & & & \\
\hline $\mathrm{N} 2$ & $86(19.8)$ & & & & & & \\
\hline N3 & $52(12.0)$ & & & & & & \\
\hline Histological stage & & 1.135 & 0.934-1.379 & 0.204 & & & \\
\hline Well & $60(13.8)$ & & & & & & \\
\hline Moderate & $208(47.9)$ & & & & & & \\
\hline Poor & $166(38.2)$ & & & & & & \\
\hline
\end{tabular}

${ }^{a}$ Cox regression model. ${ }^{b}$ Log-rank test. ${ }^{c}$ Samples are divided into two groups based on the median age at diagnosis of 65 years. HR, hazard ratio; ASPM, abnormal spindle-like microcephaly; T, tumor; N, node.

(Tables III and IV). Both univariate and multivariate Cox regression analyses revealed that age, $\mathrm{pT}$ stage, $\mathrm{pN}$ stage and ASPM expression were significant prognostic factors for OS (Table III). In TCGA cohort, only pT stage and pN stage were significant prognostic factors for OS, in addition to ASPM expression (Table IV).

Clinical specimens confirm that high ASPM expression is associated with a poor prognosis in patients with LUAD. Subsequently, to investigate the expression pattern of ASPM protein in LUAD, IHC staining we performed to analyze ASPM protein expression in 94 paired tissues of LUAD collected from patients at Qilu Hospital (Fig. 2A-D). IHC staining revealed that ASPM protein was localized in the cytoplasm. A scoring system indicated that ASPM protein expression was significantly higher in LUAD tissues than in adjacent normal tissues (Fig. 2E). Similarly, western blotting confirmed that ASPM protein expression was increased in eight paired LUAD tissues compared with in their adjacent non-tumor tissues (Fig. 2G and H). The associations between ASPM expression and clinicopathological parameters are presented in Table V, indicating that ASPM expression was significantly associated with pathological lymph node $(\mathrm{pN})$ status $(\mathrm{P}=0.041)$, TNM stage $(\mathrm{P}=0.027)$ and survival status $(\mathrm{P}=0.001)$. However, ASPM expression was not significantly associated with sex, age, pT status, pathological metastasis (pM) status and pathological stage (Table V). Additionally, Kaplan-Meier curve analysis demonstrated a significant negative association between ASPM expression and OS ( $\mathrm{P}=0.0011$; Fig. 2F).

ASPM promotes carcinogenesis of LUAD in vitro and in vivo. Considering that high ASPM expression was associated with poor survival outcomes in patients with LUAD, the function of high ASPM expression in the biological behavior of LUAD cells was further investigated. The baseline expression levels of endogenous ASPM were evaluated in a panel of six cell lines (HBE, HCC827, A549, H157, H1299 and H1975) using RT-qPCR and western blotting (Fig. S1A and B). The H1299 cell line demonstrated the highest endogenous ASPM expression and was thus selected as the cell model for ASPM-knockdown. The ASPM gene was knocked down using RNA interference (RNAi) by introducing two targeted siRNAs (siRNA1 and siRNA2) in H1299 cells. The knockdown efficiency was verified using RT-qPCR, revealing that both siRNAs significantly knocked down 
Table IV. Univariate and multivariate Cox regression analysis of different prognostic factors in patients with lung adenocarcinoma from The Cancer Genome Atlas database.

\begin{tabular}{|c|c|c|c|c|c|c|c|}
\hline \multirow[b]{2}{*}{ Characteristics } & \multirow[b]{2}{*}{ Sample, n (\%) } & \multicolumn{3}{|c|}{ Univariate analysis ${ }^{\mathrm{a}}$} & \multicolumn{3}{|c|}{ Multivariate analysis ${ }^{\mathrm{a}}$} \\
\hline & & HR & $95 \% \mathrm{CI}$ & P-value ${ }^{b}$ & HR & $95 \% \mathrm{CI}$ & P-value ${ }^{b}$ \\
\hline $\mathrm{Age}^{\mathrm{d}}$ & & 1.086 & $0.774-1.523$ & 0.635 & & & \\
\hline$<66$ & $162(48.1)$ & & & & & & \\
\hline$\geq 66$ & $175(51.9)$ & & & & & & \\
\hline $\operatorname{Sex}$ & & 0.923 & $0.660-1.292$ & 0.641 & & & \\
\hline Male & $166(49.3)$ & & & & & & \\
\hline Female & $171(50.7)$ & & & & & & \\
\hline ASPM expression & & 1.926 & $1.364-2.718$ & $<0.001$ & 1.725 & $1.219-2.441$ & 0.002 \\
\hline Low expression & $168(49.9)$ & & & & & & \\
\hline High expression & $169(50.1)$ & & & & & & \\
\hline TNM stage & & 1.590 & $1.360-1.860$ & $<0.001$ & 1.235 & $0.969-1.574$ & 0.088 \\
\hline I & $172(51)$ & & & & & & \\
\hline II & $83(24.6)$ & & & & & & \\
\hline III & $61(18.1)$ & & & & & & \\
\hline IV & $21(6.2)$ & & & & & & \\
\hline T stage & & 1.617 & $1.330-1.967$ & $<0.001$ & 1.310 & $1.05-1.635$ & 0.017 \\
\hline $\mathrm{T} 1$ & $102(30.3)$ & & & & & & \\
\hline $\mathrm{T} 2$ & $189(56.1)$ & & & & & & \\
\hline $\mathrm{T} 3$ & $28(8.3)$ & & & & & & \\
\hline $\mathrm{T} 4$ & $18(5.3)$ & & & & & & \\
\hline $\mathrm{N}$ stage & & 1.804 & $1.473-2.208$ & $<0.001$ & 1.346 & $1.024-1.769$ & 0.033 \\
\hline No & $212(62.9)$ & & & & & & \\
\hline $\mathrm{N} 1$ & $72(21.4)$ & & & & & & \\
\hline $\mathrm{N} 2+\mathrm{N} 3$ & $53(15.7)$ & & & & & & \\
\hline M stage & & 1.849 & $1.042-3.283$ & 0.036 & & & $\mathrm{NA}^{\mathrm{c}}$ \\
\hline M0 & $316(93.8)$ & & & & & & \\
\hline M1 & $21(6.2)$ & & & & & & \\
\hline
\end{tabular}

${ }^{a}$ Cox regression model. ${ }^{b}$ Log-rank test. ${ }^{c}$ Since M1 completely overlapped with Stage IV, the P-value could not be calculated in Cox regres-

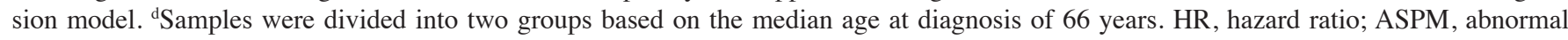
spindle-like microcephaly; T, tumor; N, node; M, metastasis; NA, not available.

ASPM expression (Fig. 3A). CCK-8 and EDU assays were used to monitor cell proliferation, and the results indicated that LUAD cells with silenced ASPM exhibited significantly impaired proliferation (Fig. 3B and C). Additionally, ASPM deficiency significantly attenuated wound healing ability compared with that in control cells (Fig. 3D). Moreover, ASPM silencing significantly suppressed migration and invasion of H1299 cells (Fig. 3D). To validate whether ASPM silencing affected epithelial-mesenchymal transition (EMT) in LUAD cells, western blotting was performed. The current results revealed that ASPM-silenced cells exhibited increased protein expression levels of the epithelial marker E-cadherin, while the expression levels of the mesenchymal marker proteins $\mathrm{N}$-cadherin and Snail were decreased compared with those in the control group (Figs. 3E and S2). Overall, the present results suggested that ASPM may exert a crucial role in the proliferation, migration and invasion of LUAD cells.
Subsequently, animal experiments were performed to evaluate the oncogenic effect of ASPM. Stable ASPM-knockdown cells (H1299 and A549) were established via lentiviral transfection using a shRNA against ASPM, and RT-qPCR results indicated that shASPM significantly inhibited ASPM expression in both cell lines (Fig. S1C and D). H1299 cells were injected subcutaneously to establish the xenograft tumor model in nude mice (Fig. 4A). The mice that were transplanted with ASPM-knockdown H1299 cells exhibited a significant inhibition of tumor growth and reduction in tumor weight (Fig. 4B and C). Furthermore, the depletion of ASPM decreased the expression levels of Ki67 and N-cadherin, and increased those of E-cadherin compared with the control group (Fig. 4D). Additionally, metastasis experiments in BALB/c nude mice were performed using shASPM-transfected H1299 cells. H1299 cells were injected into BALB/c nude mice through the caudal vein, and metastasis lesions were evaluated 7 weeks after injection (Fig. 4E). The results revealed 

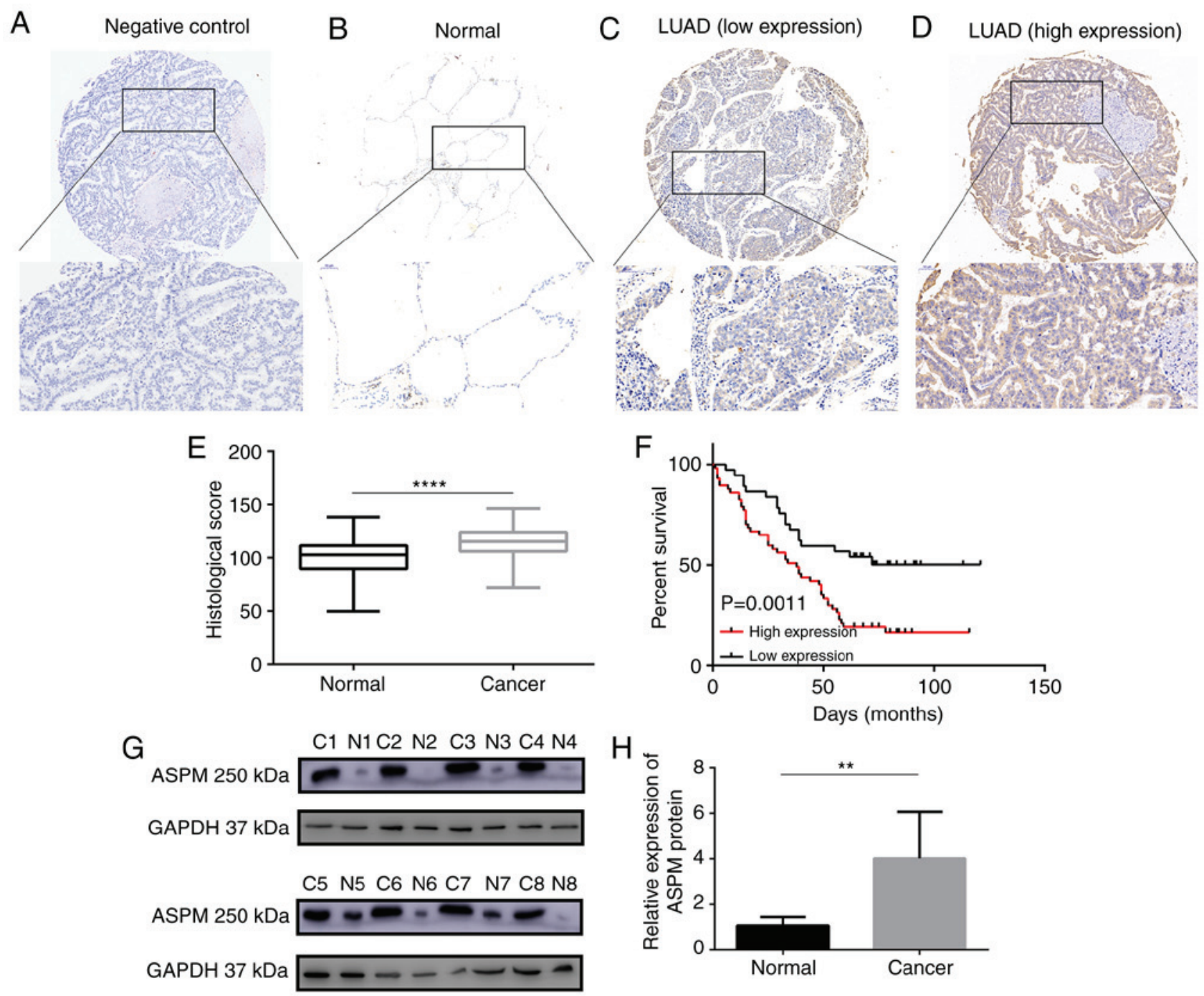

Figure 2. Clinical specimens confirm that high ASPM expression is associated with an unfavorable prognosis in patients with LUAD. Representative IHC images (magnifications, $\mathrm{x} 50$ and $\mathrm{x} 500$ ) of ASPM expression in (A) negative control (IgG), (B) normal para-tumor tissues, and LUAD tissues with (C) low ASPM expression and (D) high ASPM expression. (E) IHC score for ASPM expression in the tissue microarray. (F) Kaplan-Meier analysis of LUAD cohorts based on predictive survival analysis. (G) ASPM protein expression was detected by western blotting in 8 paired LUAD tissue samples. (H) Statistical analysis of western blotting data. ${ }^{* *} \mathrm{P}<0.01 ;{ }^{* * * *} \mathrm{P}<0.0001$. LUAD, lung adenocarcinoma; ASPM, abnormal spindle-like microcephaly; IHC, immunohistochemistry.

that ASPM-knockdown significantly decreased the number of metastatic lesions in the lung (Fig. 4F). Tumor metastasis was confirmed using hematoxylin and eosin staining (Fig. 4G). Therefore, both in vitro and in vivo data supported the proliferative and metastatic effects of ASPM in LUAD.

ASPM-knockdown suppresses the PI3K/AKT signaling pathway. In order to explore the molecular mechanism of ASPM in LUAD, transcriptome sequencing was performed in shASPM-transfected H1299 and control cells. KEGG analysis indicated that ASPM-knockdown had important effects on genes that were mainly associated with the PI3K/AKT, mitogen-activated protein kinase (MAPK), Ras-proximate-1 (Rap1), Wnt, Hippo and stem cell signaling pathways, as well as with cell adhesion molecules (CAMs) and extracellular matrix (ECM)-receptor interaction (Fig. 5A). GO revealed genes involved in 'extracellular matrix organization' (GO:0030198), 'collagen-containing extracellular matrix' (GO:0062023), 'anchored component of membrane' (GO:0031225) and 'extracellular matrix binding' (GO:0050840) (Fig. 5C). It is well known that activation of the PI3K/AKT signaling pathway can lead to EMT in cancer and that the extracellular matrix organization is altered to promote metastasis during EMT $(31,32)$. Therefore, it was hypothesized that ASPM promoted LUAD metastasis by activating EMT via the PI3K/AKT signaling pathway. Western blotting results further proved that ASPM-knockdown led to increased protein expression levels of the epithelial marker E-cadherin and decreased protein expression levels of the mesenchymal marker proteins $\mathrm{N}$-cadherin and Snail, and molecules in PI3K signaling pathway (PI3K and pAKT) in both H1299 and A549 cells (Figs. 5B and S3).

Activation of the PI3K/AKT signaling pathway can restore the migration and invasion of shASPM-transfected LUAD cells. The role of the PI3K/AKT signaling pathway in LUAD metastasis caused by ASPM was further investigated. A specific activator of the PI3K/AKT signaling pathway, 740 Y-P, was 
Table V. Association between ASPM expression and clinicopathological characteristics in patients with lung adenocarcinoma $(n=94)$.

ASPM expression

\begin{tabular}{|c|c|c|c|c|}
\hline & & & & \\
\hline Characteristics & All cases, $\mathrm{n}(\%)$ & Low expression $(n=37)$ & High expression $(n=57)$ & P-value ${ }^{a}$ \\
\hline Sex & & & & 0.786 \\
\hline Male & $53(56.4)$ & 22 & 31 & \\
\hline Female & $41(43.6)$ & 15 & 26 & \\
\hline Age $^{b}$ & & & & 0.304 \\
\hline$<60$ & $43(45.7)$ & 14 & 29 & \\
\hline$\geq 60$ & $51(54.3)$ & 23 & 28 & \\
\hline pT status & & & & 0.762 \\
\hline $\mathrm{T} 1$ & $20(21.3)$ & 8 & 12 & \\
\hline $\mathrm{T} 2$ & $50(53.2)$ & 21 & 29 & \\
\hline $\mathrm{T} 3$ & $18(19.1)$ & 7 & 11 & \\
\hline $\mathrm{T} 4$ & $6(6.4)$ & 1 & 5 & \\
\hline pN status & & & & 0.041 \\
\hline No & $42(44.7)$ & 21 & 21 & \\
\hline N1 & $17(18.1)$ & 8 & 9 & \\
\hline $\mathrm{N} 2+\mathrm{N} 3$ & $35(37.2)$ & 8 & 27 & \\
\hline pM status & & & & $>0.999$ \\
\hline M0 & $93(98.9)$ & 37 & 56 & \\
\hline M1 & $1(1.1)$ & 0 & 1 & \\
\hline TNM stage & & & & 0.027 \\
\hline I & $30(31.9)$ & 16 & 14 & \\
\hline II & $20(21.3)$ & 10 & 19 & \\
\hline III+IV & $44(46.8)$ & 11 & 33 & \\
\hline Pathological stage & & & & 0.280 \\
\hline $\mathrm{I}$ & $11(11.7)$ & 6 & 5 & \\
\hline II & $52(55.3)$ & 17 & 35 & \\
\hline III & $31(33.0)$ & 14 & 17 & \\
\hline Event & & & & 0.001 \\
\hline Alive & $29(30.9)$ & 19 & 10 & \\
\hline Dead & $65(69.1)$ & 18 & 47 & \\
\hline
\end{tabular}

aPearson's $\chi^{2}$ test or Fisher's exact test was used for statistical analysis. ${ }^{\mathrm{b}}$ Age was divided based on the median age at diagnosis of 60 years. ASPM, abnormal spindle-like microcephaly; pT, pathological tumor; pN, pathological lymph node; pM, pathological metastasis.

used to activate PI3K activity after ASPM-knockdown. Consequently, ASPM-knockdown inhibited EMT (leading to decreased $\mathrm{N}$-cadherin and Snail expression, and increased E-cadherin expression) in H1299 and A549 cells, whereas 740 Y-P treatment partially restored these changes (Figs. 6A and S4). Moreover, 740 Y-P treatment significantly reversed the inhibition of H1299 and A546 cell invasion and migration induced by ASPM-knockdown (Fig. 6B and C), suggesting that the PI3K/AKT signaling pathway was the key effector of the ASPM-mediated invasion and migration.

\section{Discussion}

In the present study, the clinical and oncogenic role of ASPM in LUAD was explored. LUAD is one of the deadliest tumors worldwide (33). Due to the lack of effective diagnostic and prognostic evaluation biomarkers, the morbidity and mortality rates in patients with LUAD are on the rise $(33,34)$. Identifying new molecular markers that can detect recurrence and metastasis, guide prognosis and improve survival rates in patients with LUAD are urgently required. Although some studies reported a polygene model that included the ASPM-predicted prognosis in $\operatorname{LUAD}(35,36)$, to the best of our knowledge the present study was the first to employ the GEO database as a discovery cohort and TCGA as a validation cohort to address the role of ASPM in LUAD. The current study revealed that ASPM mRNA expression was upregulated in LUAD tissues, and the upregulation of ASPM expression independently predicted a poor prognosis in patients with LUAD. Furthermore, higher ASPM protein 

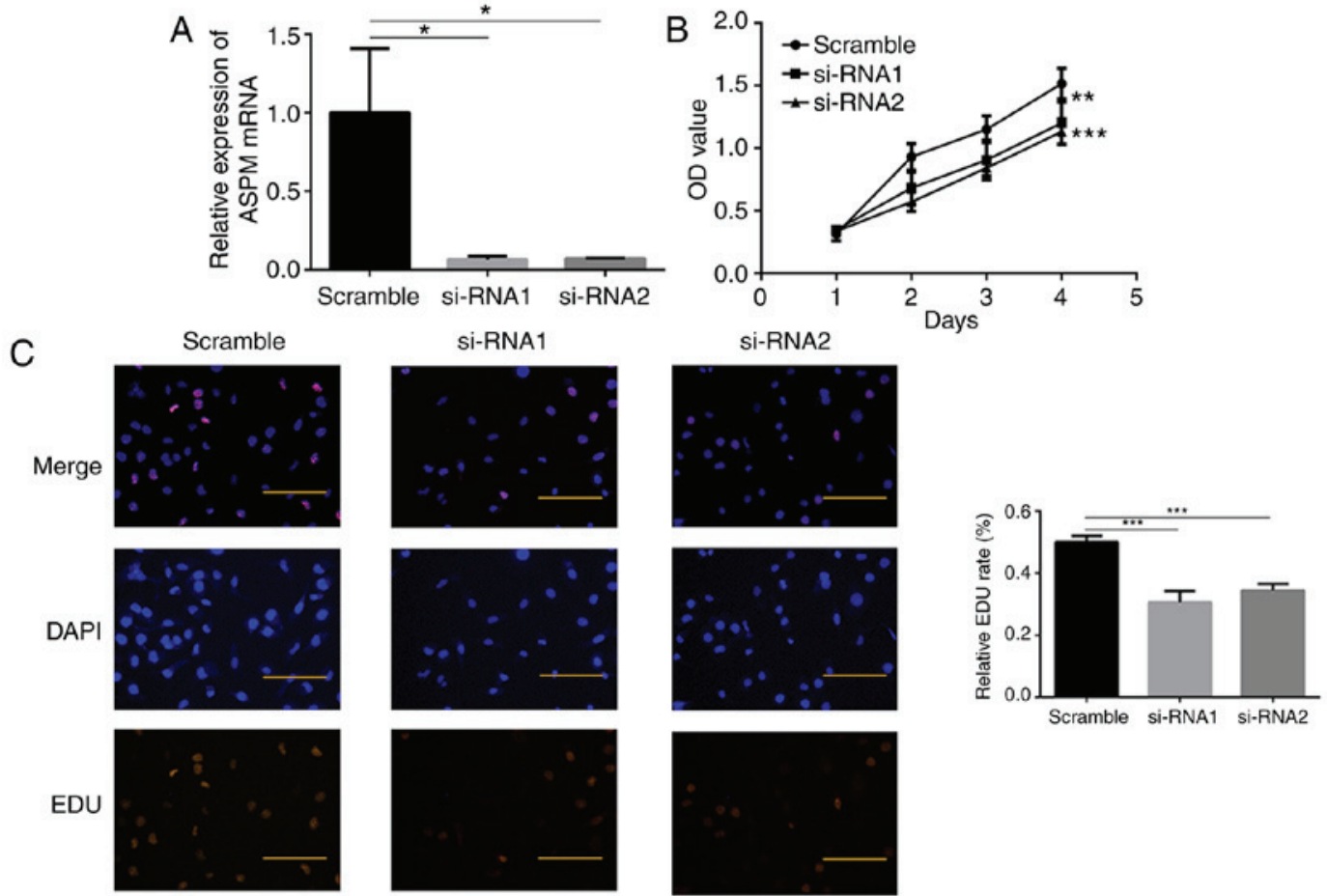

D

Scramble

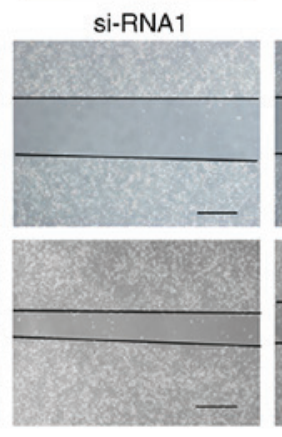

si-RNA2
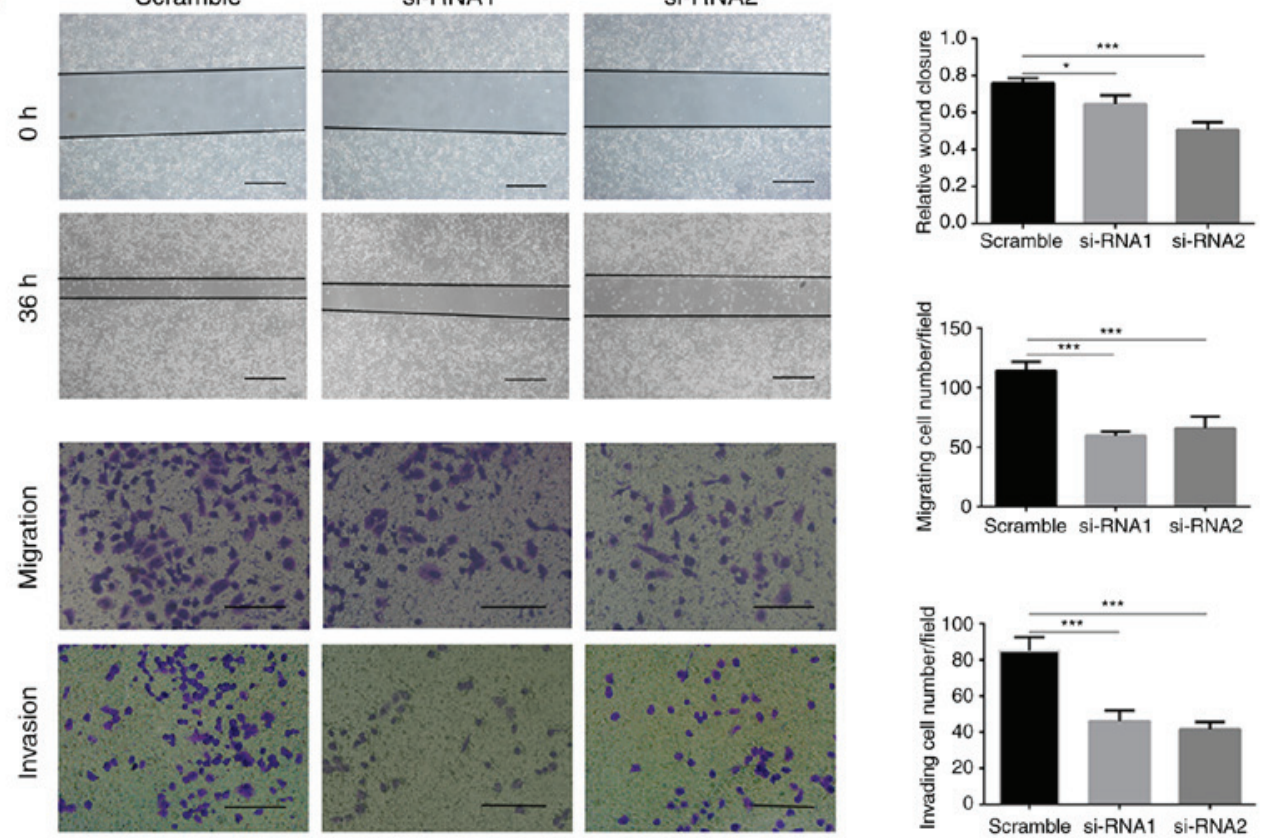

$\mathrm{E}$
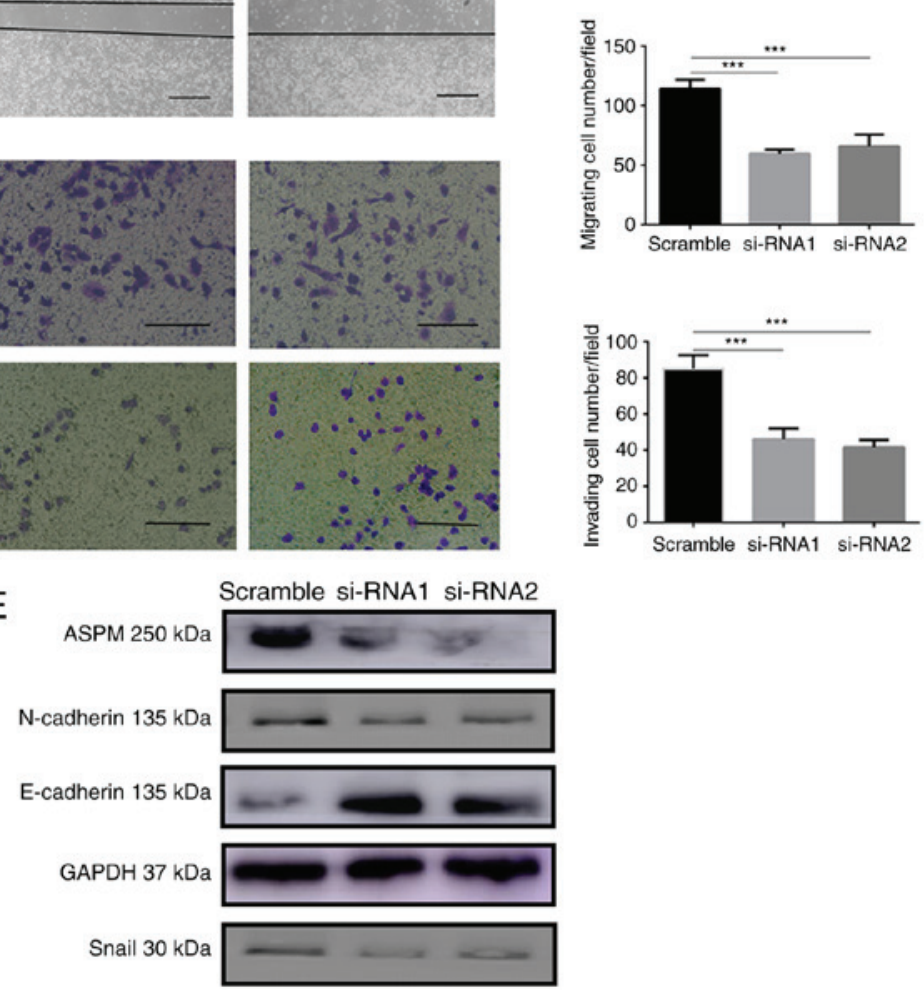

Figure 3. ASPM promotes lung adenocarcinoma cell proliferation, migration and invasion in vitro. (A) Reverse transcription-quantitative PCR was used to confirm ASPM-knockdown using two siRNAs in H1299 cells. (B) Cell Counting Kit-8 and (C) EDU assays were performed to identify proliferation after ASPM-knockdown in H1299 cells (scale bar, $100 \mu \mathrm{m}$ ). (D) Wound healing (scale bar, $100 \mu \mathrm{m}$ ), migration and invasion (scale bar, $50 \mu \mathrm{m}$ ) assays were performed to identify the migratory and invasive abilities after ASPM-knockdown in H1299 cells. (E) Changes in the expression levels of the epithelial-mesenchymal transition biomarkers E-cadherin, N-cadherin and Snail after ASPM-knockdown were detected by western blotting. Data are presented as the mean \pm SD $(\mathrm{n}=3) .{ }^{*} \mathrm{P}<0.05,{ }^{* *} \mathrm{P}<0.01$ and ${ }^{* * * *} \mathrm{P}<0.001$ vs. scramble. ASPM, abnormal spindle-like microcephaly; si-RNA, small interfering RNA; OD, optical density; EDU, 5-Ethynyl-2'-deoxyuridine. 
A

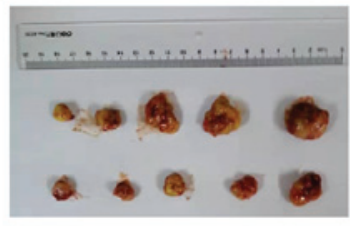

E

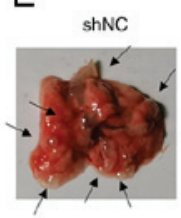

B

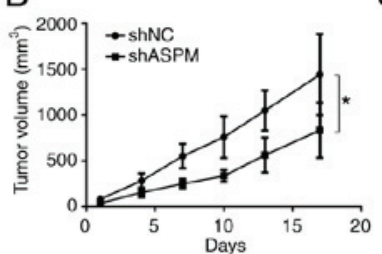

C

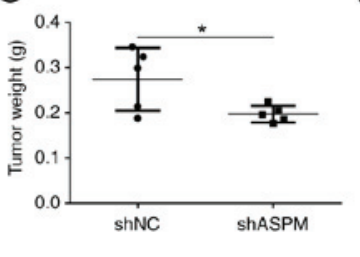

D

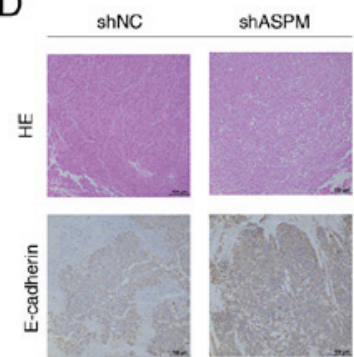

G

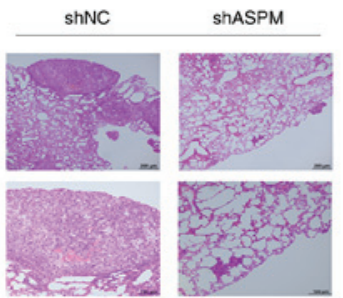

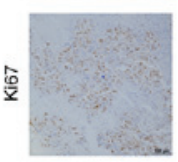
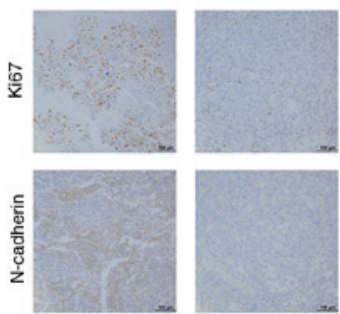

Figure 4. ASPM promotes lung adenocarcinoma cell proliferation and metastasis in vivo. (A) Representative images of tumors from different treatment groups 5 weeks after tumor cell injections. (B) Tumor volume and (C) weight were lower for xenograft tumors with ASPM-knockdown than for xenograft tumors with shNC. (D) Representative images of immunohistochemical staining for Ki67, E-cadherin and N-cadherin in xenograft tumors (magnification, x100), (E) Representative images of lung metastasis. (F) Bar chart of lung metastasis nodules in shNC and shASPM groups. (G) Representative images of HE staining of lung metastasis in shNC and shASPM groups (magnification, $\mathrm{x} 100$ and $\mathrm{x} 200$ ). $\mathrm{P}<0.05 ;{ }^{* *} \mathrm{P}<0.01$. ASPM, abnormal spindle-like microcephaly; sh, short hairpin RNA; NC, negative control; HE, hematoxylin and eosin.
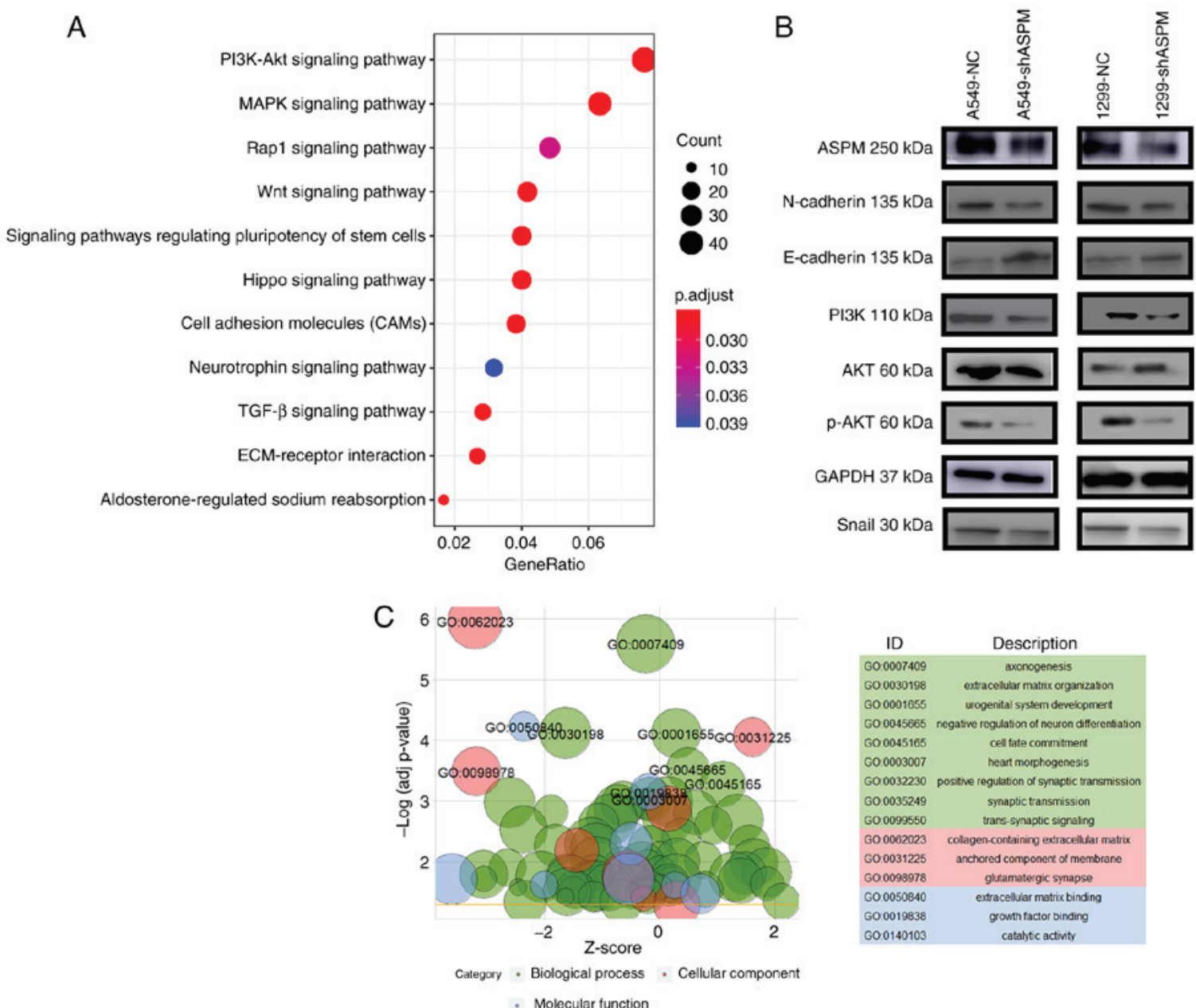

- Molecular function

Figure 5. ASPM-knockdown suppresses the PI3K/AKT signaling pathway. (A) Kyoto Encyclopedia of Genes and Genomes pathway analysis and (C) GO analysis after ASPM-knockdown in H1299 cells identified from transcriptome sequencing. Statistical significance is indicated by different colors. The size of the circle represents the number of genes in each pathway. (B) Protein expression levels of components of the PI3K/AKT signaling pathway and EMT biomarkers following ASPM-knockdown. ASPM, abnormal spindle-like microcephaly; sh, short hairpin RNA; NC, negative control; GO, Gene Ontology; p, phosphorylated. 


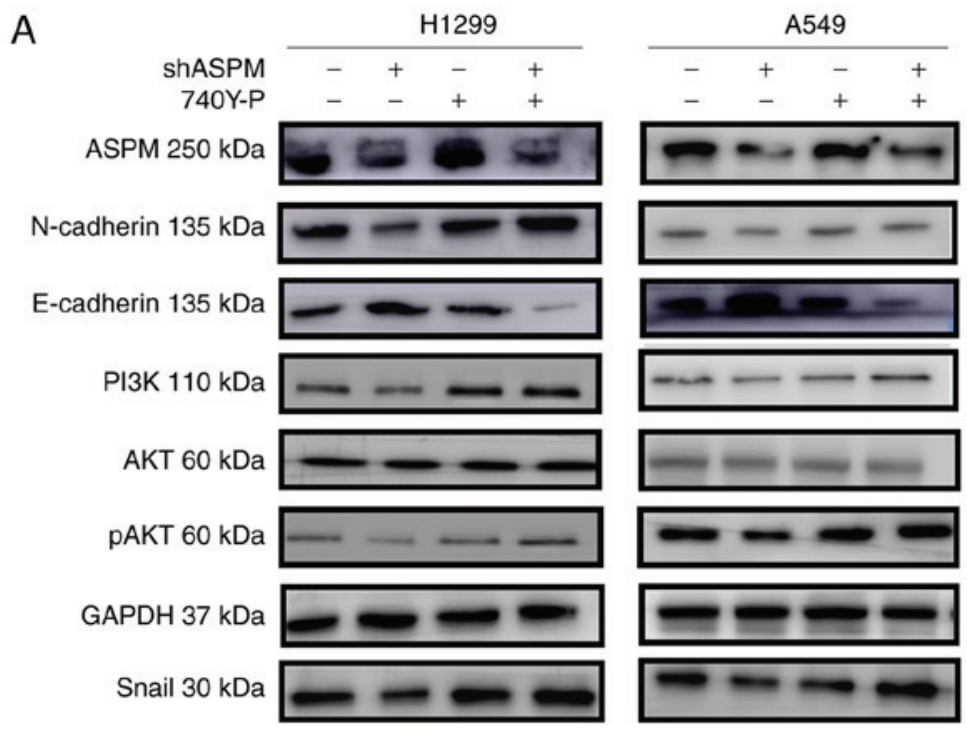

B

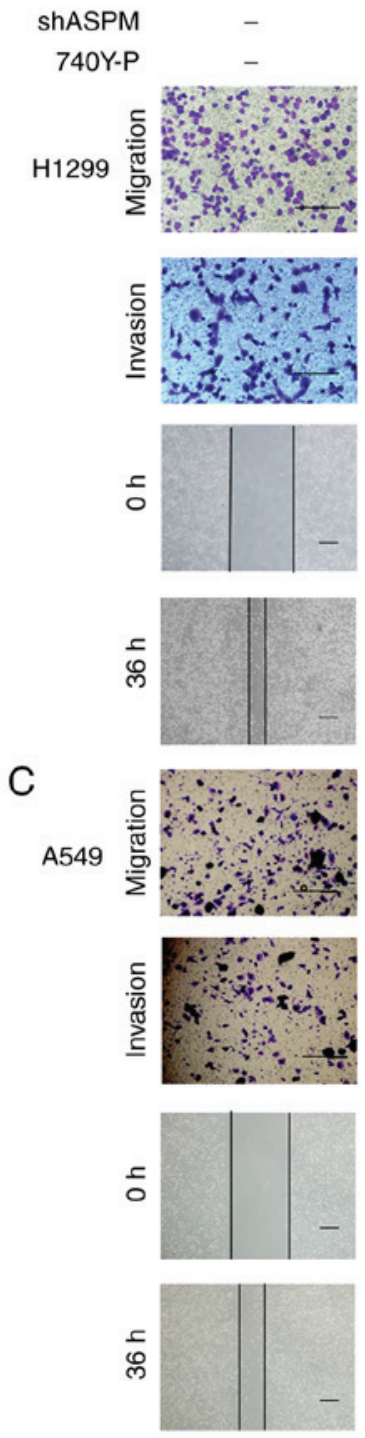

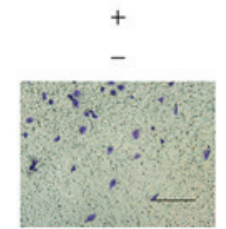
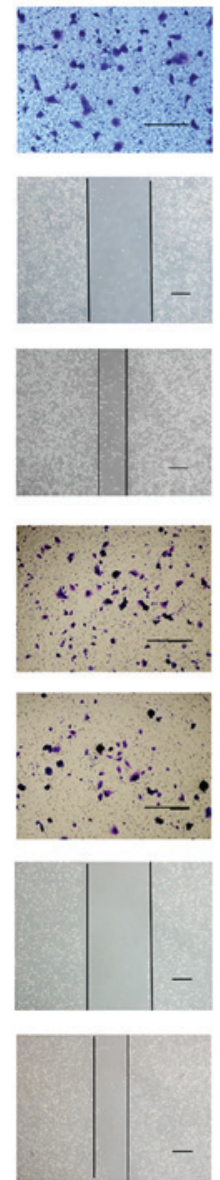
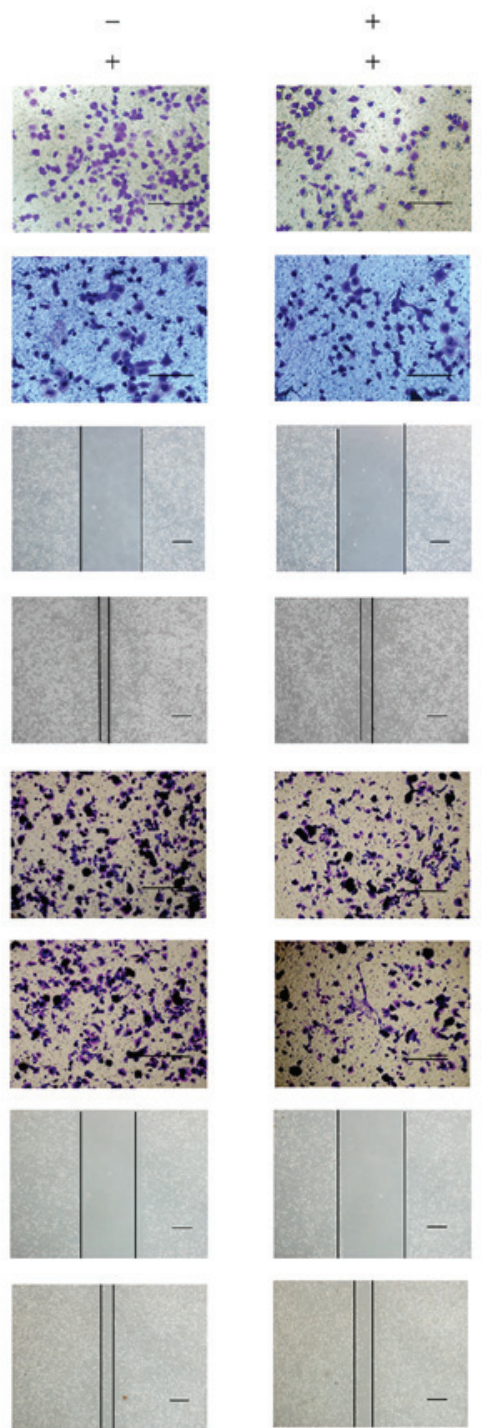
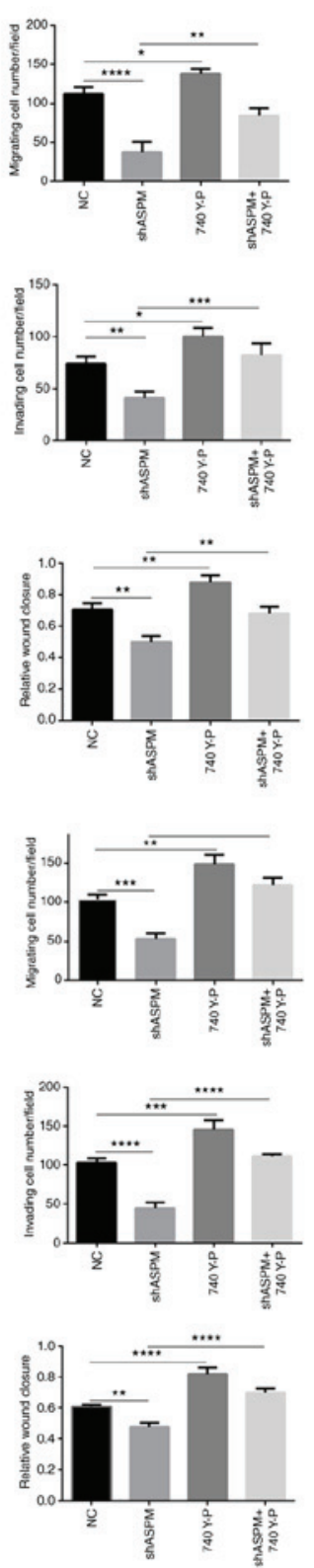

Figure 6. Activation of the PI3K/AKT signaling pathway restores the migratory and invasive abilities of shASPM-transfected lung adenocarcinoma cells. (A) Changes in epithelial-mesenchymal transition biomarker expression, including N-cadherin, E-cadherin and Snail, were detected in ASPM-knockdown H1299 and A549 cells with or without 740 Y-P treatment for $24 \mathrm{~h}$. Wound healing (scale bar, $100 \mu \mathrm{m}$ ), migration and invasion assays (scale bar, $50 \mu \mathrm{m}$ ) in ASPM-knockdown (B) H1299 and (C) A549 cells with or without 740 Y-P treatment for 24 h. Data are presented as the mean \pm SD $(n=3) .{ }^{~} \mathrm{P}<0.05$; ${ }^{* * *} \mathrm{P}<0.01$; ${ }^{* * * *} \mathrm{P}<0.001 ;{ }^{* * * *} \mathrm{P}<0.0001$. ASPM, abnormal spindle-like microcephaly; sh, short hairpin RNA; NC, negative control; p, phosphorylated. 
expression in LUAD was associated with disease progression, indicating that ASPM expression may be used as a potential biomarker. Similarly, ASPM expression is elevated in multiple types of cancer, including bladder cancer, pancreatic cancer (37), ovarian cancer (15), breast cancer (38), endometrial cancer (39), glioblastoma $(12,40)$, hepatocellular carcinoma (16) and lung squamous carcinoma (41), where it confers a poor prognosis.

A series of in vitro and in vivo assays were performed in the present study to illustrate the biological function of ASPM in regulating LUAD cell proliferation and migration. ASPM-knockdown markedly attenuates the proliferation and migration of cells in prostate cancer (13), endometrial cancer (39), pancreatic cancer (37), glioma (40) and lung squamous carcinoma (41). Overall, the findings of the current study indicated that ASPM served a critical role in LUAD cell proliferation and migration.

Accumulating evidence has revealed that EMT participates in the invasion and early metastasis in numerous types of tumor by inducing the expression of mesenchymal markers and decreasing that of epithelial markers $(42,43)$. A number of key transcription factors, including Twist, Snail and Slug, have been considered as major drivers of the EMT program (31). In the present study, ASPM-knockdown had a significant negative influence on EMT by suppressing the expression levels of mesenchymal markers (N-cadherin and Snail) and inducing the expression levels of the epithelial marker E-cadherin. Additionally, in vivo assays indicated upregulation of E-cadherin expression and downregulation of N-cadherin expression in the ASPM-knockdown group. Thus, ASPM-knockdown may affect LUAD cell migration and invasion by interfering with the EMT process.

The activation of EMT depends on a number of oncogenic signaling pathways, including the intracellular PI3K/AKT signaling pathway (44). The current results demonstrated that the PI3K/AKT signaling pathway was associated with ASPM-induced EMT. A previous study suggested that the activation of the PI3K/AKT signaling pathway led to LUAD tumorigenesis, including increased cell proliferation, metastasis and drug resistance (45). Phosphorylated AKT can regulate pivotal EMT genes by inducing key transcription factors, including Snail, that affect the progression of cancer (44). The present results indicated that ASPM-knockdown affected the expression levels of PI3K and phosphorylated AKT. Moreover, the attenuation effect of ASPM-knockdown on LUAD cell migration and invasion, and the ASPM-induced EMT was markedly restored when the PI3K/AKT signaling pathway was activated using 740 Y-P. Therefore, the mechanism of ASPM-induced promotion of EMT may be mediated through the activation of the PI3K/AKT signaling pathway, ultimately driving LUAD cell migration and invasion. However, the specific mechanisms by which ASPM activates PI3K/AKT should be further explored. As initial suggestions for possible mechanisms, the current RNA-sequencing results revealed that ASPM-knockdown had important effects on genes that are mainly associated with the MAPK, Rapl, Wnt, Hippo and stem cell signaling pathways, as well as CAMs and ECM-receptor interaction. This is in agreement with previous studies indicating that ASPM is involved in the regulation of cancer stem cells and promotes $\mathrm{Wnt}$ pathway activity by stabilizing the Dvl-3 protein $(8,46,47)$. To the best of our knowledge, the present study was the first to demonstrate that ASPM promoted activation of the PI3K/AKT signaling pathway. However, a direct association between ASPM and the PI3K/AKT signaling pathway was not observed. Future studies should focus on the mechanism by which ASPM activates the PI3K/AKT signaling pathway and the association between ASPM and other signaling pathways, including MAPK, Rap1 and Hippo.

In conclusion, the current study first demonstrated that ASPM was highly expressed in LUAD tissues and was an independent biomarker for predicting prognosis in patients with LUAD. ASPM-knockdown in LUAD inhibited proliferation, migration and invasion in LUAD cells. Furthermore, functional and mechanistic studies revealed that ASPM regulated EMT to promote LUAD metastasis through the activation of the PI3K/AKT signaling pathway. Therefore, the current findings provided a theoretical basis for the biological function of ASPM in LUAD and indicated that ASPM may serve as a clinically relevant biomarker and a potential therapeutic target in LUAD.

\section{Acknowledgements}

Not applicable.

\section{Funding}

The present study was supported by the National Natural Science Foundation of China (grant nos. 81802397 and 81672292) and the Taishan Scholar Program of Shandong Province (grant no. ts201712087).

\section{Availability of data and materials}

The datasets used and/or analyzed during the current study are available from the corresponding author on reasonable request. The datasets generated and/or analyzed during the current study (GSE140797, GSE19804, GSE116959, GSE31210 and GSE68465) are available in the GEO repository (GSE140797, https://www.ncbi.nlm.nih.gov/geo/query/acc. cgi?acc=GSE140797; GSE19804, https://www.ncbi.nlm.nih. gov/gds/?term=GSE19804; GSE116959, https://www.ncbi. nlm.nih.gov/gds/?term=GSE116959; GSE31210, https://www. ncbi.nlm.nih.gov/gds/?term=GSE31210; and GSE68465, https://www.ncbi.nlm.nih.gov/gds/?term=GSE68465)

\section{Authors' contributions}

JW and HT designed the study. JW, JL, HL, JH, JJ, YL, ZF and RZ performed the experiments. JW and JL performed the statistical analysis. JW wrote the manuscript and HT revised the manuscript. JW and JL are responsible for confirming the authenticity of the data. All authors read and approved the final manuscript.

\section{Ethics approval and consent to participate}

All experiments were approved and supervised by the Medical Ethics Committee of Qilu Hospital of Shandong University 
(Jinan, China; approval no. KYLL-2016-097). Patients provided written informed consent for the use of their samples in the study. All animal experiments were approved by the Medical Ethics Committee of Shandong University (approval no. KYLL-2016-097).

\section{Patient consent for publication}

Not applicable.

\section{Competing interests}

The authors declare that they have no competing interests.

\section{References}

1. Bray F, Ferlay J, Soerjomataram I, Siegel RL, Torre LA and Jemal A: Global cancer statistics 2018: GLOBOCAN estimates of incidence and mortality worldwide for 36 cancers in 185 countries. CA Cancer J Clin 68: 394-424, 2018.

2. Cancer Genome Atlas Research Network: Comprehensive molecular profiling of lung adenocarcinoma. Nature 511: 543-550, 2014.

3. Hirsch FR, Scagliotti GV, Mulshine JL, Kwon R, Curran WJ Jr, Wu YL and Paz-Ares L: Lung cancer: Current therapies and new targeted treatments. Lancet 389: 299-311, 2017.

4. Borczuk AC: Prognostic considerations of the new world health organization classification of lung adenocarcinoma. Eur Respir Rev 25: 364-371, 2016.

5. Zhong X, Liu L, Zhao A, Pfeifer GP and Xu X: The abnormal spindle-like, microcephaly-associated (ASPM) gene encodes a centrosomal protein. Cell Cycle 4: 1227-1229, 2005.

6. Kouprina N, Pavlicek A, Mochida GH, Solomon G, Gersch W, Yoon YH, Collura R, Ruvolo M, Barrett JC, Woods CG, et al Accelerated evolution of the ASPM gene controlling brain size begins prior to human brain expansion. PLoS Biol 2: E126, 2004.

7. Passemard S, Titomanlio L, Elmaleh M, Afenjar A, Alessandri JL, Andria G, Billette de Villemeur T, Boespflug-Tanguy $O$ Burglen L, Del Giudice E, et al: Expanding the clinical and neuroradiologic phenotype of primary microcephaly due to ASPM mutations. Neurology 73: 962-969, 2009.

8. Capecchi MR and Pozner A: ASPM regulates symmetric stem cell division by tuning Cyclin E ubiquitination. Nat Commun 6 : 8763,2015

9. Gai M, Bianchi FT, Vagnoni C, Verni F, Bonaccorsi S, Pasquero S, Berto GE, Sgrò F, Chiotto AA, Annaratone L, et al: ASPM and CITK regulate spindle orientation by affecting the dynamics of astral microtubules. EMBO Rep 18: 1870, 2017.

10. Jiang K, Rezabkova L, Hua S, Liu Q, Capitani G, Altelaar AFM, Heck AJR, Kammerer RA, Steinmetz MO and Akhmanova A: Microtubule minus-end regulation at spindle poles by an ASPM-katanin complex. Nat Cell Biol 19: 480-492, 2017.

11. An X, Huang Y and Zhao P: Expression of ASPM in colonic adenocarcinoma and its clinicopathologic significance. Int J Clin Exp Pathol 10: 8968-8973, 2017.

12. Chen X, Huang L, Yang Y, Chen S, Sun J, Ma C, Xie J, Song Y and Yang J: ASPM promotes glioblastoma growth by regulating G1 restriction point progression and Wnt- $\beta$-catenin signaling. Aging (Albany NY) 12: 224-241, 2020.

13. Xie JJ, Zhuo YJ, Zheng Y, Mo RJ, Liu ZZ, Li BW, Cai ZD, Zhu XJ, Liang YX, He HC and de Zhong W: High expression of ASPM correlates with tumor progression and predicts poor outcome in patients with prostate cancer. Int Urol Nephrol 49: 817-823, 2017.

14. Timaner M and Shaked Y: Elucidating the roles of ASPM isoforms reveals a novel prognostic marker for pancreatic cancer. J Pathol 250: 123-125, 2020.

15. Alsiary R, Bruning-Richardson A, Bond J, Morrison EE, Wilkinson N and Bell SM: Deregulation of microcephalin and ASPM expression are correlated with epithelial ovarian cancer progression. PLoS One 9: e97059, 2014.

16. Lin SY, Pan HW, Liu SH, Jeng YM, Hu FC, Peng SY, Lai PL and Hsu HC: ASPM is a novel marker for vascular invasion, early recurrence, and poor prognosis of hepatocellular carcinoma. Clin Cancer Res 14: 4814-4820, 2008.
17. Xu Z, Zhang Q, Luh F, Jin B and Liu X: Overexpression of the ASPM gene is associated with aggressiveness and poor outcome in bladder cancer. Oncol Lett 17: 1865-1876, 2019.

18. Chansky K, Detterbeck FC, Nicholson AG, Rusch VW, Vallieres E, Groome P, Kennedy C, Krasnik M, Peake M, Shemanski L, et al: The IASLC lung cancer staging project: External validation of the revision of the TNM stage groupings in the eighth edition of the TNM classification of lung cancer. J Thorac Oncol 12: 1109-1121, 2017.

19. Liang J, Li H, Han J, Jiang J, Wang J, Li Y, Feng Z, Zhao R, Sun Z, Lv B and Tian H: Mex3a interacts with LAMA2 to promote lung adenocarcinoma metastasis via PI3K/AKT pathway. Cell Death Dis 11: 614, 2020.

20. Lu TP, Tsai MH, Lee JM, Hsu CP, Chen PC, Lin CW, Shih JY, Yang PC, Hsiao CK, Lai LC and Chuang EY: Identification of a novel biomarker, SEMA5A, for non-small cell lung carcinoma in nonsmoking women. Cancer Epidemiol Biomarkers Prev 19: 2590-2597, 2010

21. Moreno Leon L, Gautier M, Allan R, Ilie M, Nottet N, Pons N, Paquet A, Lebrigand K, Truchi M, Fassy J, et al: The nuclear hypoxia-regulated NLUCAT1 long non-coding RNA contributes to an aggressive phenotype in lung adenocarcinoma through regulation of oxidative stress. Oncogene 38: 7146-7165, 2019.

22. Okayama H, Kohno T, Ishii Y, Shimada Y, Shiraishi K, Iwakawa R, Furuta K, Tsuta K, Shibata T, Yamamoto S, et al: Identification of genes upregulated in ALK-positive and EGFR/KRAS/ALK-negative lung adenocarcinomas. Cancer Res 72: 100-111, 2012.

23. Director's Challenge Consortium for the Molecular Classification of Lung Adenocarcinoma; Shedden K, Taylor JM, Enkemann SA, Tsao MS, Yeatman TJ, Gerald WL, Eschrich S, Jurisica I, Giordano TJ, et al: Gene expression-based survival prediction in lung adenocarcinoma: A multi-site, blinded validation study. Nat Med 14: 822-827, 2008

24. Huang da W, Sherman BT and Lempicki RA: Systematic and integrative analysis of large gene lists using DAVID bioinformatics resources. Nat Protoc 4: 44-57, 2009.

25. Huang da W, Sherman BT and Lempicki RA: Bioinformatics enrichment tools: Paths toward the comprehensive functional analysis of large gene lists. Nucleic Acids Res 37: 1-13, 2009.

26. Yeo W, Chan SL, Mo FK, Chu CM, Hui JW, Tong JH, Chan AWH, Koh J, Hui EP, Loong H, et al: Phase I/II study of temsirolimus for patients with unresectable hepatocellular carcinoma (HCC)-a correlative study to explore potential biomarkers for response. BMC Cancer 15: 395, 2015.

27. Azim HA Jr, Peccatori FA, Brohee S, Branstetter D, Loi S, Viale G, Piccart M, Dougall WC, Pruneri G and Sotiriou C: RANK-ligand (RANKL) expression in young breast cancer patients and during pregnancy. Breast Cancer Res 17: 24, 2015.

28. Su D, Liao Z, Feng B, Wang T, Shan B, Zeng Q, Song J and Song Y: Pulsatilla chinensis saponins cause liver injury through interfering ceramide/sphingomyelin balance that promotes lipid metabolism dysregulation and apoptosis. Phytomedicine 76: 153265, 2020.

29. Shan B, Ai Z, Zeng S, Song Y, Song J, Zeng Q, Liao Z, Wang T, Huang $\mathrm{C}$ and $\mathrm{Su}$ D: Gut microbiome-derived lactate promotes to anxiety-like behaviors through GPR81 receptor-mediated lipid metabolism pathway. Psychoneuroendocrinology 117: 104699, 2020.

30. Livak KJ and Schmittgen TD: Analysis of relative gene expression data using real-time quantitative PCR and the 2(-Delta Delta C(T)) method. Methods 25: 402-408, 2001.

31. Larue L and Bellacosa A: Epithelial-mesenchymal transition in development and cancer: Role of phosphatidylinositol 3 kinase/AKT pathways. Oncogene 24: 7443-7454, 2005.

32. Paolillo M and Schinelli S: Extracellular matrix alterations in metastatic processes. Int J Mol Sci 20: 4947, 2019.

33. Siegel RL, Miller KD and Jemal A: Cancer statistics, 2020. CA Cancer J Clin 70: 7-30, 2020.

34. Oudkerk M, Liu S, Heuvelmans MA, Walter JE and Field JK: Lung cancer LDCT screening and mortality reduction-evidence, pitfalls and future perspectives. Nat Rev Clin Oncol 18: 135-151, 2021.

35. Li Z, Qi F and Li F: Establishment of a gene signature to predict prognosis for patients with lung adenocarcinoma. Int J Mol Sci 21: 8479, 2020.

36. Li L, Peng M, Xue W, Fan Z, Wang T, Lian J, Zhai Y, Lian W, Qin D and Zhao J: Integrated analysis of dysregulated long non-coding RNAs/microRNAs/mRNAs in metastasis of lung adenocarcinoma. J Transl Med 16: 372, 2018. 
37. Hsu CC, Liao WY, Chan TS, Chen WY, Lee CT, Shan YS, Huang PJ, Hou YC, Li CR and Tsai KK: The differential distributions of ASPM isoforms and their roles in Wnt signaling, cell cycle progression, and pancreatic cancer prognosis. J Pathol 249: 498-508, 2019.

38. Tang J, Lu M, Cui Q, Zhang D, Kong D, Liao X, Ren J, Gong Y and Wu G: Overexpression of ASPM, CDC20, and TTK confer a poorer prognosis in breast cancer identified by gene co-expression network analysis. Front Oncol 9: 310, 2019.

39. Zhou JW, Wang H, Sun W, Han NN and Chen L: ASPM is a predictor of overall survival and has therapeutic potential in endometrial cancer. Am J Transl Res 12: 1942-1953, 2020.

40. Bikeye SN, Colin C, Marie Y, Vampouille R, Ravassard P, Rousseau A, Boisselier B, Idbaih A, Calvo CF, Leuraud P, et al: ASPM-associated stem cell proliferation is involved in malignant progression of gliomas and constitutes an attractive therapeutic target. Cancer Cell Int 10: 1, 2010.

41. Yuan YJ, Sun Y, Gao R, Yin ZZ, Yuan ZY and Xu LM: Abnormal spindle-like microcephaly-associated protein (ASPM) contributes to the progression of lung squamous cell carcinoma (LSCC) by regulating CDK4. J Cancer 11: 5413-5423, 2020.

42. Santamaria PG, Moreno-Bueno G, Portillo F and Cano A: EMT: Present and future in clinical oncology. Mol Oncol 11: 718-738, 2017.
43. Aiello NM and Kang Y: Context-dependent EMT programs in cancer metastasis. J Exp Med 216: 1016-1026, 2019.

44. Karimi Roshan M, Soltani A, Soleimani A, Rezaie Kahkhaie K, Afshari AR and Soukhtanloo M: Role of AKT and mTOR signaling pathways in the induction of epithelial-mesenchymal transition (EMT) process. Biochimie 165: 229-234, 2019.

45. Papadimitrakopoulou V: Development of PI3K/AKT/mTOR pathway inhibitors and their application in personalized therapy for non-small-cell lung cancer. J Thorac Oncol 7: 1315-1326, 2012.

46. Wang F, Li J, Liu J and Zhao Q: Controversial role of the possible oxyntic stem cell marker ASPM in gastric cancer. J Pathol 241: 559-561, 2017.

47. Pai VC, Hsu CC, Chan TS, Liao WY, Chuu CP, Chen WY, Li CR, Lin CY, Huang SP, Chen LT and Tsai KK: ASPM promotes prostate cancer stemness and progression by augmenting Wnt-Dvl-3- $\beta$-catenin signaling. Oncogene 38: 1340-1353, 2019.

(i) $\Theta$ This work is licensed under a Creative Commons BY NO ND Attribution-NonCommercial-NoDerivatives 4.0 International (CC BY-NC-ND 4.0) License. 